\title{
Implementación de una planta productora de grasa lubricante de disulfuro de molibdeno para el sector minero
}

\author{
José Antonio Sastre Noboa \\ Universidad de Lima \\ Ingeniería Industrial n²9, 2011, ISSN 1025-9929, pp. 183-206 \\ Recibido: 18 de abril del 2011 / Aprobado: 2 de mayo del 2011
}

\begin{abstract}
Resumen: Este estudio busca mostrar el potencial que tiene el Perú para brindar un valor agregado a las materias primas que se extraen en el país. Específicamente se trata de la molibdenita, que es utilizada como lubricante sólido, en aleaciones, en grasas y otras aplicaciones; además, provee una gran variedad de valiosos subproductos. Cabe destacar que a pesar de que el Perú es el cuarto productor de este mineral en el mundo, aún no cuenta con plantas especializadas para su tratamiento.
\end{abstract}

Palabrasclave: molibdeno / molibdenita / grasas lubricantes / proyectos industriales / empresas industriales / industria de lubricantes / minería / Perú

\section{Installation of a production plant for lubricant grease with molybdenum's suldife for the mining companies}

\begin{abstract}
This study is a sample of the potential that lies in Peru to give value-added products instead of raw material. Specifically molybdenite, which is used as a solid lubricant, alloys, greases and other applications; besides, it provides a wide variety of byproducts. It is important to remark that Peru is the fourth country supplier of this mineral worldwide and nowadays specialized plants for its treatment are absent nationally.

Keywords: molybdenum / molybdenite / lubricants greases / industrial projects / industrial companies / lubricant industry / minerals / mining / Peru
\end{abstract}




\section{MARCO TEÓRICO}

El disulfuro de molibdeno, también llamado molibdenita, es extraído de yacimientos geológicos de tipo porfirítico, de los cuales también se extrae cobre, en una pureza de entre 70 y 90 por ciento.

Las dos terceras partes de la producción mundial de este concentrado se derivan a la aleación de metales y a la elaboración de ferromolibdeno, el resto es utilizado en la industria de los lubricantes.

El disulfuro de molibdeno es un lubricante sólido que soporta altas temperaturas y reduce significativamente la fricción. Esto se debe a que el molibdeno es el segundo metal conocido que soporta las mayores temperaturas, superado solamente por el tungsteno, y a las propiedades que proporciona frente a la fricción, según explica Mundi (1972):

La molécula de disulfuro de molibdeno tiene una estructura cristalina de tipo laminar u "hojaldrada" que presenta coeficientes muy pequeños de fricción en presencia de altas velocidades, o bien a bajas velocidades y altas cargas, esto se debe a la facilidad de deslizamiento entre sí de las laminillas. $[\ldots]$

El poder adherente del metal mencionado se basa en la existencia de valencias libres de los átomos de azufre y la disminución del coeficiente de fricción frente a las cargas altas en la automática orientación plano paralela de sus laminillas en la dirección de las cargas; el poder de "deslizamiento" del disulfuro de molibdeno disminuye ligera y lentamente después de un largo tiempo de trabajo en condiciones excesivamente duras.

Por lo tanto, la grasa lubricante con disulfuro de molibdeno es utilizada en máquinas con alta fricción o altas temperaturas. Además, hay máquinas en las que solamente se puede utilizar este tipo de grasa por los altos niveles de fricción que presentan. En otros países se consume esta grasa, con distinto porcentaje de disulfuro, en diversos equipos, como armas, vehículos, entre otros.

En nuestro país, las empresas mineras nacionales se encuentran en constante crecimiento y generan altos índices de producción, lo que hace necesario que mantengan sus equipos en el mejor estado posible, para lo cual consumen más grasa; pero actualmente, la producción nacional de este producto es muy limitada y solamente se cuenta con una empresa que lo elabora. Esto obliga a su importación, principalmente de Estados Unidos. 


\section{ESTUDIO DE MERCADO}

\subsection{Definición del producto}

El producto materia de este artículo es una grasa lubricante con un porcentaje de disulfuro de molibdeno, lo cual le agrega mayor eficiencia y funciones óptimas en el uso industrial. Es utilizado en máquinas con rodamientos que presentan alta fricción a bajas velocidades y soporta altas temperaturas de trabajo.

Se comercializa en barriles de 400 libras y el principal mercado son las empresas mineras, por ser las que más consumen este producto, ya que sus equipos requieren un buen agente lubricante para evitar su deterioro; se debe tener en cuenta que una parada de planta por mantenimiento, sea reactivo o planificado, conlleva una gran pérdida monetaria para este sector.

La venta y publicidad de este producto se realiza directamente con el cliente, dado que se quiere lograr un vínculo directo con este a fin de poder generar alianzas estratégicas, considerando que los proveedores del disulfuro de molibdeno son, a su vez, clientes, y mantener una mayor lealtad por parte de estos. El mercado planteado presenta dificultades de ingreso, por tal motivo se requiere que la fuerza de ventas se oriente a atender directamente a los clientes y a brindarles un buen valor agregado.

A los clientes se les brindará un producto de menor costo frente a los productos importados; especial, según los requerimientos de las empresas; y se ofrecerá la asesoría de un experto en tribología ${ }^{1}$ para atender todas las consultas de los usuarios, además de capacitar a su personal de mantenimiento en caso de que sea solicitado.

\subsection{Análisis de la demanda}

El proyecto busca desplazar las importaciones de este producto a fin de brindarle mayor fortaleza al mercado nacional. Teniendo esto en

1 Tribología es la ciencia que estudia la fricción, el desgaste y la lubricación que tiene lugar durante el contacto entre superficies sólidas en movimiento. 
cuenta, se analizaron sus importaciones históricas, filtrando la partida de lubricantes por producto, y se realizó una proyección de la demanda total del proyecto utilizando esta información.

Dado que realizar encuestas a las empresas mineras presenta dificultades, se realizaron tres juicios de expertos para determinar la participación del proyecto dentro de la demanda total. De esta manera se pudo establecer el porcentaje de las importaciones a ser desplazado a lo largo de la vida útil del proyecto. En el cuadro 1 se muestra la información planteada y el programa de ventas.

Cuadro 1

Programa de ventas para la vida útil del proyecto

\begin{tabular}{cccc}
\hline Año & $\begin{array}{c}\text { Participación } \\
\text { en el mercado } \\
(\%)\end{array}$ & $\begin{array}{c}\text { Demanda } \\
\text { proyectada }(\mathbf{k g})\end{array}$ & $\begin{array}{c}\text { Ventas } \\
\text { anuales } \mathbf{( k g )}\end{array}$ \\
\hline 2011 & 13,0 & $795.647,0$ & $103.434,1$ \\
2012 & 13,0 & $814.321,0$ & $105.861,7$ \\
2013 & 14,5 & $831.396,0$ & $120.552,4$ \\
2014 & 16,0 & $847.151,0$ & $135.544,2$ \\
2015 & 17,5 & $861.794,0$ & $150.814,0$ \\
2016 & 19,0 & $875.488,0$ & $166.342,7$ \\
2017 & 22,0 & $888.361,0$ & $195.439,4$ \\
2018 & 22,0 & $900.515,0$ & $198.113,3$ \\
2019 & 22,0 & $912.035,0$ & $200.647,7$ \\
2020 & 22,0 & $922.990,0$ & $203.057,8$ \\
\hline
\end{tabular}

Elaboración propia.

\subsection{Análisis de los precios}

Por los motivos planteados en párrafos anteriores se analizaron los precios internacionales, con el objeto de determinar el precio de venta del producto presentado en el proyecto. De esta manera, se revisaron sus importaciones históricas y se consideró su precio CIF. Luego se obtuvo la tendencia de estos precios, pero por las variaciones habidas en los últimos tres años, esta resultó en una ecuación lineal con valores muy elevados; por ello, tuvo que ser corregida a fin de brindar el valor que más se aproximara al precio de venta actual. Finalmente, se pro- 
yectó el precio de venta para la vida útil del proyecto, que se presenta en el cuadro 2. Este precio se encuentra 26 centavos de dólar por debajo del precio internacional por kilogramo, para poder brindar una ventaja competitiva frente a los productos importados.

\section{Cuadro 2}

Proyección del precio de venta del producto

\begin{tabular}{cccc}
\hline Año & $\begin{array}{c}\text { Precio } \\
\text { internacional }(\mathbf{\$})\end{array}$ & $\begin{array}{c}\text { Precio } \\
\text { proyecto }(\$)\end{array}$ & $\begin{array}{c}\text { Precio } \\
\text { proyecto }(\mathbf{S} / .)\end{array}$ \\
\hline 2011 & 7,72 & 7,46 & 21,63 \\
2012 & 8,18 & 7,92 & 22,97 \\
2013 & 8,64 & 8,38 & 24,30 \\
2014 & 9,10 & 8,84 & 25,64 \\
2015 & 9,56 & 9,30 & 26,97 \\
2016 & 10,02 & 9,76 & 28,30 \\
2017 & 10,48 & 10,22 & 29,64 \\
2018 & 10,94 & 10,68 & 30,97 \\
2019 & 11,40 & 11,14 & 32,31 \\
2020 & 11,86 & 11,60 & 33,64 \\
\hline
\end{tabular}

Fuente: <www.aduanet.gob.pe>.

Elaboración propia.

\section{DISPONIBILIDAD DE INSUMOS}

A continuación se presenta una descripción breve de los insumos requeridos para la elaboración de la grasa lubricante con disulfuro de molibdeno y se realiza el análisis de la participación del proyecto dentro de la producción nacional del disulfuro de molibdeno, que es el insumo más relevante para dicho análisis.

\subsection{Aceite base}

El aceite base significa el 94\% del peso total de la grasa; sin embargo, las características principales de la grasa están dadas por los aditivos que esta contenga. Existen tres tipos principales de aceite base: sintético, mineral y vegetal. 
El aceite base mineral de base parafínica, utilizado en el presente proyecto, es estable a altas temperaturas y tiene buena resistencia a la oxidación; además, es de bajo costo y de fácil adquisición.

\subsection{Espesante}

El espesante es requerido para aumentar la adhesividad de la grasa a las superficies metálicas, con el fin de evitar que sean desplazadas con facilidad; además, retienen los fluidos por absorción. Este insumo es uno de los elementos con mayor influencia en las características de la grasa, y forma una estructura fibrosa que contiene aceite; existen dos categorías: jabones convencionales y jabones compuestos.

En este proyecto se utilizará un jabón complejo de litio, que es muy eficaz. Con este espesante las grasas se vuelven más resistentes a la pérdida de consistencia y a las fugas, adquieren excelentes propiedades anticorrosivas y selladoras, así como una moderada resistencia al agua; asimismo, provee un mejor funcionamiento de los aditivos que otras grasas, posee una extraordinaria estabilidad estructural y térmica, y tolera temperaturas de trabajo de hasta $175^{\circ} \mathrm{C}$ al tener un elevado punto de goteo $\left(260^{\circ} \mathrm{C}\right)$ (Aranzabe y Málaga 2004).

\subsection{Disulfuro de molibdeno}

El concentrado de disulfuro de molibdeno $\left(\mathrm{MoS}_{2}\right)$ es un polvo apolar (hidrofóbico) de color negro, proviene de los yacimientos mineros nacionales y es extraído como tal; posee aproximadamente un $90 \%$ de disulfuro de molibdeno, por lo que requiere de un proceso de flotación selectiva para elevar este porcentaje hasta $97 \%$. El disulfuro de molibdeno ocupa un $3 \%$ de la mezcla total de la grasa a elaborar. Es el material que brinda las cualidades más importantes a la grasa, como el antidesgaste.

\subsection{Aditivos para la grasa}

Una vez elaborada la grasa desde un inicio se va a requerir de tres aditivos. En primer lugar, un inhibidor de corrosión, el cual suspende la corrosión de las superficies metálicas si esta ya se ha originado o la evita en caso de que, debido a condiciones ambientales, se pueda presentar; se utiliza sulfonato de amoniaco. En segundo lugar, un agente EP (presión extrema), el cual aumenta la capacidad de la grasa de so- 
portar los choques y vibraciones comunes en los arranques y paradas de las máquinas; se utiliza naftenato de plomo. Y en tercer lugar, un inhibidor de oxidación, el cual impide la oxidación y descomposición de la grasa; se utiliza fenil-alfa-naftilamino o fenil-beta-naftilamino. Asimismo, es posible que se requieran algunos aditivos adicionales para funciones específicas.

\subsection{Aditivos de flotación}

Los reactivos necesarios para la flotación dependen de los componentes en el concentrado. A partir de los manuales de concentración de minerales y de los informes de flotación de disulfuro de molibdeno se ha podido determinar que los reactivos necesarios son: cal (2 kg por t) como modificador de $\mathrm{pH}$; cianuro de sodio ( $1 \mathrm{~kg}$ por $\mathrm{t}$ ) como depresor de $\mathrm{Cu}$ y modificador de $\mathrm{pH}$; xantato isopopílico de sodio Z-11 (200 g por t) como colector aniónico, y aceite de pino (150 g por t) como espumante (Alcalá 2006, Tantaleán 2004: 83).

\subsection{Participación del proyecto dentro de la disponibilidad de insumos}

El disulfuro de molibdeno es extraído en el Perú por tres compañías mineras: Southern Copper Corporation (Cuajone y Toquepala, Moquegua y Tacna, respectivamente), Antamina (San Marcos, Huari, Ancash) y Cerro Verde (Arequipa); y viene con una concentración en peso de disulfuro de molibdeno de 90,31\%.

Utilizando la producción histórica de este mineral en los yacimientos mencionados, el programa de ventas proyectado y considerando que la grasa utiliza $3 \%$ de este insumo a una concentración de $97 \%$ en peso, se ha podido determinar el requerimiento de disulfuro de molibdeno para el proyecto y su participación dentro de la producción nacional. En el cuadro 3 se muestra el resultado del análisis realizado. 


\section{Cuadro 3}

Participación del proyecto dentro de la producción de disulfuro de molibdeno en Perú

\begin{tabular}{cccccc}
\hline Año & $\begin{array}{c}\text { Producción } \\
\mathbf{( k g )}\end{array}$ & $\begin{array}{c}\text { Ventas } \\
\text { grasa } \\
\mathbf{( k g )}\end{array}$ & $\begin{array}{c}\text { Necesidad } \\
\text { MoS2 } \mathbf{3} \% \mathbf{3}] \\
\mathbf{( k g )}\end{array}$ & $\begin{array}{c}\text { Necesidad total } \\
\text { concentrado } \\
\mathbf{( k g )}\end{array}$ & $\begin{array}{c}\text { Participación } \\
\%\end{array}$ \\
\hline 2011 & 17.600 .400 & 103.434 & 3.103 & 3.598 & 0,02 \\
2012 & 17.959 .531 & 105.862 & 3.176 & 3.682 & 0,02 \\
2013 & 18.290 .714 & 120.552 & 3.617 & 4.193 & 0,02 \\
2014 & 18.598 .391 & 135.544 & 4.066 & 4.715 & 0,03 \\
2015 & 18.885 .994 & 150.814 & 4.524 & 5.246 & 0,03 \\
2016 & 19.156 .236 & 166.343 & 4.990 & 5.786 & 0,03 \\
2017 & 19.411 .300 & 195.439 & 5.863 & 6.798 & 0,04 \\
2018 & 19.652 .970 & 198.113 & 5.943 & 6.891 & 0,04 \\
2019 & 19.882 .725 & 200.648 & 6.019 & 6.979 & 0,04 \\
2020 & 20.101 .802 & 203.058 & 6.092 & 7.063 & 0,04 \\
\hline
\end{tabular}

En el cuadro 4 se muestra la lista de insumos requeridos en el proyecto y su costo.

\section{Cuadro 4}

Costo de los insumos

\begin{tabular}{lc}
\hline \multicolumn{1}{c}{ Materia prima } & $\begin{array}{c}\text { "Precio } \\
\text { (US } \mathbf{~ / ~ k g ) " ~}\end{array}$ \\
\hline Disulfuro de molibdeno & 15,63 \\
Jabón de litio & 98,20 \\
Aceite base & 1,03 \\
Sulfonato de amoniaco & 0,52 \\
Naftenato de plomo & 152,00 \\
Fenil-Alfa-naftilamino & 433,00 \\
Cal & 0,03 \\
Cianuro de sodio & 6,04 \\
Aceite de pino & 1,75 \\
Xantato Isopropílico Sodio & 0,78 \\
\hline
\end{tabular}

Elaboración propia. 


\section{INGENIERÍA DEL PROYECTO}

\subsection{Definición del producto sobre la base de sus características de fabricación}

El producto es una grasa lubricante, elaborada a partir de aceite base mineral parafínico, el cual es mezclado mediante un proceso de mezcla homogénea junto con jabón de litio en polvo (agente espesante), disulfuro de molibdeno pulverizado con un tamaño promedio de 0,75 micrones por partícula (agente espesante y antidesgaste), sulfonato de amoniaco (inhibidor de corrosión), naftenato de plomo (agente de extrema presión) y fenil-alfa-naftilamino (inhibidor de oxidación). Esta grasa es de color negro, con olor tenue, una densidad de $0,87 \mathrm{~kg} / \mathrm{l}$ y un punto de goteo aproximado de $180^{\circ} \mathrm{C}$.

En el cuadro 5 se muestra la composición en peso de la grasa lubricante con disulfuro de molibdeno.

\section{Cuadro 5}

Composición de la grasa lubricante con disulfuro de molibdeno

\begin{tabular}{lcc}
\hline \multicolumn{1}{c}{ Compuesto } & Contenido \\
\hline Aceite base sintético & $94 \%$ \\
Disulfuro de molibdeno & $3 \%$ \\
Jabón de litio & \multicolumn{2}{c}{$2 \%$} \\
Aditivos & \multicolumn{2}{c}{$1 \%$} \\
$+\quad$ Sulfonato de amoniaco & + & $96 \%$ \\
$+\quad$ Naftenato de plomo & + & $3 \%$ \\
$+\quad$ Fenil-alfa-naftilamino & + & $1 \%$ \\
\hline
\end{tabular}

Fuente: Castrol Motor y Oil, Isopetrol S. A., Lubritec S.A., Repsol YPF (2009).

Elaboración propia.

\subsection{Proceso de producción}

Se inicia con el procesamiento del concentrado de disulfuro de molibdeno, el cual es recibido, analizado para determinar su ley y almacenado esperando su entrada al proceso de concentración. El concentrado es pesado e ingresa al proceso con una concentración aproximada de 90,31\% en peso de disulfuro de molibdeno (molibdenita). 
El concentrado es agregado junto con el agua (32\% sólidos) a la celda Rougher y se añaden los reactivos de flotación: cal ( $2 \mathrm{~kg}$, modificador de $\mathrm{pH}$ ), cianuro de sodio ( $1 \mathrm{~kg}$, depresor de $\mathrm{Cu}$ ), xantato isopropílico de sodio (200 g, colector) y aceite de pino (150 g, espumante); las cantidades están especificadas para una tonelada de concentrado. Se ingresa aire comprimido a la celda por medio del agitador, para permitir la flotación del disulfuro de molibdeno; la espuma (cola) que se va formando en la parte superior de la celda Rougher va fluyendo hacia una celda vacía. Luego, en esta celda vacía (celda Cleaner) se realiza una reflotación del concentrado (agregando agua hasta conseguir un aproximado de $28,57 \%$ en peso de sólidos y nuevamente reactivos de flotación), para obtener su concentración final. El pH promedio en las celdas es de 12 y el tiempo de flotación es aproximadamente una hora por celda (incluyendo la carga y descarga).

El relave proveniente de las celdas Rougher y Cleaner es enviado a un decantador, del cual se extraen los sólidos, estos son secados al ambiente y vendidos como ganga. El agua residual pasa por un separador de grasas (las grasas son almacenadas para ser vendidas), luego por un sistema de ósmosis inversa de cinco fases y finalmente es almacenada en un tanque junto con agua de suministro (también tratada por este sistema de ósmosis inversa), a fin de proveer agua al proceso.

El concentrado proveniente de la celda Cleaner, que contiene $80 \%$ en peso de sólidos aproximadamente, y una concentración aproximada de $97,0 \%$ de disulfuro de molibdeno, es filtrado utilizando el filtro de faja al vacío, de donde sale con solo un $8 \%$ de humedad aproximada. La faja es de tela y debajo de ella se encuentran orificios conectados a una bomba de vacío, mediante la cual se extrae el agua para ser reincorporada al proceso. Ingresa aire comprimido bajo los bordes de la faja para permitirle a esta moverse, dado que la bomba de vacío la pega al metal. Finalmente, el mineral ingresa al secador rotativo con quemador de diesel.

El secador rotativo cuenta con un quemador de petróleo, con un consumo aproximado de $0,9 \mathrm{gal} / \mathrm{h}$ según el proveedor del equipo, y calienta el mineral hasta $300^{\circ} \mathrm{C}$ para extraer la humedad que este mantiene, permitiendo la salida del mineral con tan solo $1,5 \%$ de humedad, generándose gas de combustión como residuo. A continuación, el mineral es enviado al micropulverizador. 
El micropulverizador Micronizer utiliza aire comprimido 55 SCFM (pies cúbicos estándar por minuto) para reducir las partículas de disulfuro de molibdeno hasta 0,75 micrones (medida preferente para la elaboración de la grasa). Luego el disulfuro pulverizado es depositado en un almacén de productos en proceso.

El disulfuro de molibdeno (3\%) es dosificado manualmente dentro del mezclador de paletas, junto con el jabón de litio (2\%), los aditivos (1\%) y el aceite base (94\%). Primero ingresa el aceite base y se va realizando la mezcla hasta conseguir la homogeneidad deseada; finalmente ingresa el espesante, hasta conseguir la consistencia deseada (15 minutos finales, aproximadamente). El tiempo de esta operación es de más o menos una hora, incluyendo los tiempos de carga y descarga de la máquina, 5 y 10 minutos, respectivamente.

Una vez realizado el mezclado, la grasa es envasada manualmente en barriles de 400 libras mediante dos bombas neumáticas para grasa, luego un operario coloca un tapón en el agujero secundario del barril, seguidamente la máquina selladora llena el barril con nitrógeno, un gas inerte para mantener en mejor estado la grasa; a continuación el operario coloca un tapón en el agujero primario del barril, coloca las tapas a ambos agujeros y los asegura. Finalmente, el operario imprime y pega las etiquetas al barril.

Por último, los barriles son llevados al almacén de productos terminados, en espera de su traslado final al cliente.

El proyecto consta de dos procesos definidos: la concentración del mineral y el mezclado de la grasa. Los diagramas 1 y 2 muestran el DOP y el diagrama de flujo, respectivamente, de la concentración del mineral; los diagramas 3 y 4 muestran el DOP y el diagrama de flujo, respectivamente, del mezclado de la grasa. 


\section{Diagrama 1}

DOP de la concentración del mineral

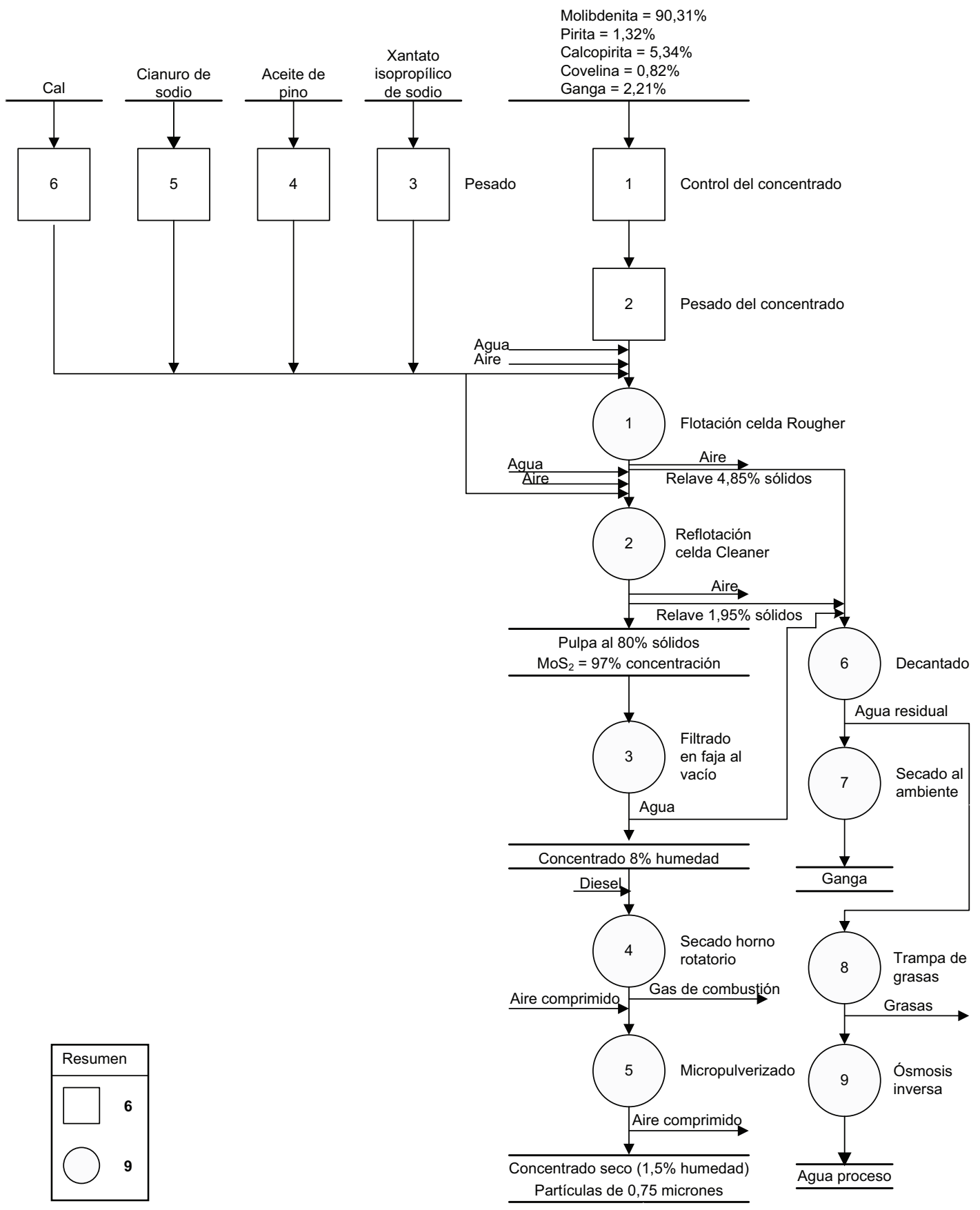

Elaboración propia. 


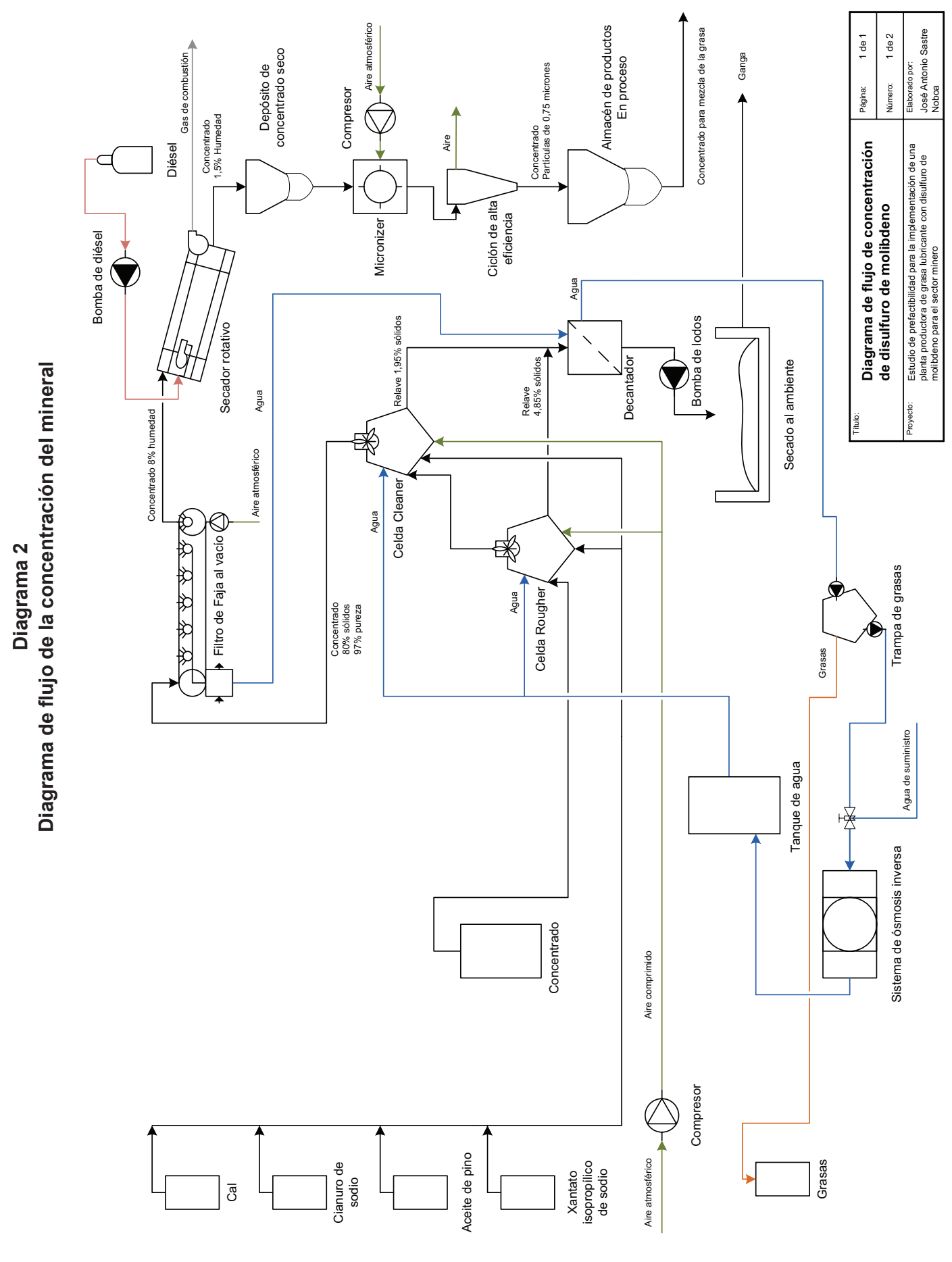

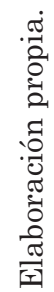


Diagrama 3

DOP del mezclado de la grasa

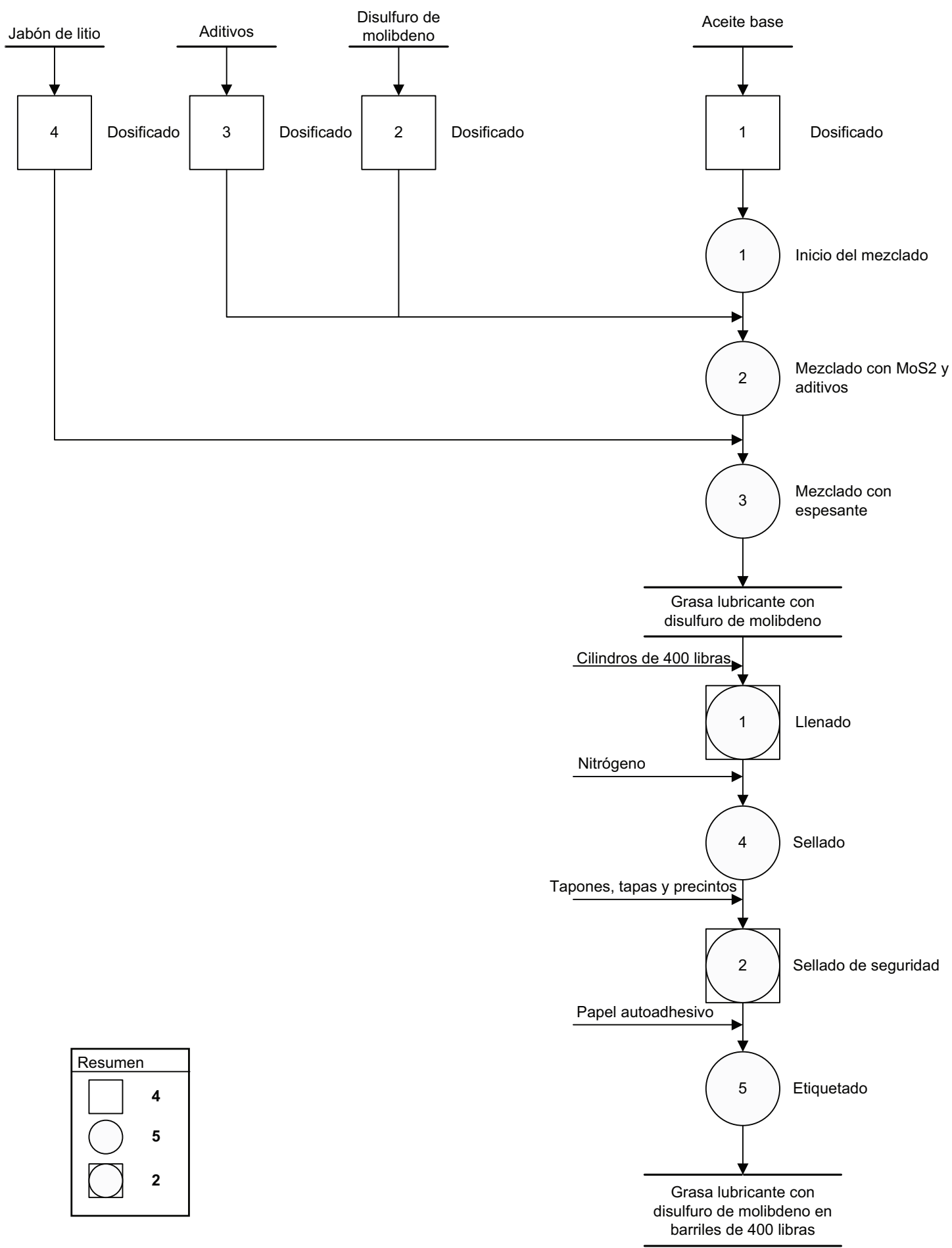

Elaboración propia. 


$$
\text { id }
$$




\section{ASPECTOS ECONÓMICOS Y FINANCIEROS}

\subsection{Inversión}

En el cuadro 6 se muestra la inversión requerida en las distintas partidas.

\section{Cuadro 6}

Inversión requerida para el proyecto

\begin{tabular}{lc}
\hline Activo fijo tangible & $\begin{array}{c}\text { Inversión requerida } \\
\text { (US\$) }\end{array}$ \\
\hline Terreno & $\$ 178.500$ \\
Obras civiles & $\$ 110.075$ \\
Muebles y enseres & $\$ 14.280$ \\
Otros activos & $\$ 5.950$ \\
Maquinaria y equipos & $\$ 306.425$ \\
Equipos de cómputo & $\$ 5.950$ \\
Total inversión requerida & $\$ 621.180$ \\
\hline & \\
\hline Activo fijo intangible & Inversión requerida \\
\hline Sistemas de información & (US) \\
\hline Total inversión requerida & $\$ 11.900$ \\
\hline & $\$ 11.900$ \\
\hline Capital de trabajo & \\
\hline Existencias & $\$ 135.575$ \\
Disponibles (caja y bancos) & Inversión requerida \\
\hline (US\$)
\end{tabular}

Elaboración propia. 


\subsection{Evaluación económica y financiera del proyecto}

Considerando un costo del capital propio igual a 11,61\% (Kallpa Securities 2010), una tasa de interés para la deuda de 14,44\% (Banco Continental 2010), y que el $20 \%$ de la inversión será aportada por capital propio se pudo realizar la evaluación del proyecto. En el cuadro 7 se muestran los indicadores obtenidos.

\section{Cuadro 7}

Indicadores para la evaluación del proyecto

\begin{tabular}{lr}
\hline Van financiero & S/. 2.541.299 \\
Relación Beneficio / Costo & 6,96 \\
Tasa interna de retorno financiera & $42 \%$ \\
Periodo de recupero (años) & 5,07 \\
\hline
\end{tabular}

Elaboración propia.

Este resultado nos demuestra que el proyecto es atractivo. El VAN es alto y la TIR es mayor que el costo promedio ponderado de capital $(10,41 \%)$.

\subsection{Análisis de sensibilidad}

Utilizando el análisis de sensibilidad unidimensional, se ha podido determinar que el proyecto puede soportar una variación en el precio o en el volumen de ventas de hasta $10,55 \%$ antes de dejar de ser atractivo para invertir.

\subsection{Impacto social}

Para poder establecer el impacto social se realizó el reporte de situación social del proyecto para su primer año de funcionamiento. Utilizando el balance general y el estado de resultados tradicional se pudo determinar el importe que será vertido a los distintos grupos de interés de la empresa (stakeholders). El resultado se muestra en el cuadro 8. 


\section{Cuadro 8}

Reporte de situación social del proyecto para el primer año

Ventas

Costos Directos:

Nacional
Importado

Valor Agregado Producido

Distribución por grupos de interes (sociales):
S/. $\quad 1.373 .303$

S/. $\quad 41.207$

S/. $\quad 709.786$

S/. $\quad 750.993$

S/. $\quad 622.310$

S/. $\quad 520.959$

S/. - -

S/. -

S/. $\quad-$

S/. -

S/. $\quad-145.073$

S/. $\quad 246.424$

S/. $\quad 622.310$

\section{INFORMACIÓN COMPLEMENTARIA:}

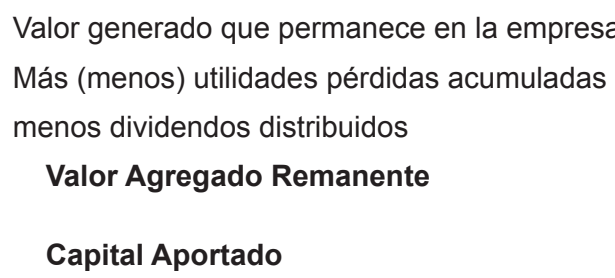

S/. $\quad 1.542 .800$

S/. $\quad-284.853$

S/. $\quad 1.257 .947$

S/. $\quad 517.044$

Porcentaje de valor agregado retenido sobre

capital invertido

$243 \%$

Valor administrado por la empresa vertido al estado
Impuestos indirectos netos
S/. $\quad-174.988$
Impuestos directos pagados
S/. -
Impuesto a la renta por dividendos
S/. -
Valor vertido al Estado
S/. $\quad-174.988$

Elaboración propia. 
De esta manera, se puede observar que el proyecto afectará, económicamente, en forma positiva a la comunidad. El primer año se vierte un valor negativo al Estado a causa del escudo fiscal obtenido como consecuencia de la adquisición de los equipos necesarios para el funcionamiento de la planta.

\section{CONCLUSIONES Y RECOMENDACIONES}

- Existen las condiciones de mercado y los requerimientos tecnológicos que permiten instalar y operar con éxito económico la planta productora de grasa lubricante a base de disulfuro de molibdeno para el sector minero.

- Es factible brindar asesoría sobre tribología y capacitación a los clientes de la empresa, y ofrecerles una grasa especial para sus requerimientos, dado el tamaño de planta y la tecnología seleccionada, brindando un producto único con un valor agregado importante.

- Es económicamente viable y rentable la instalación de la planta productora de grasa lubricante con disulfuro de molibdeno para el sector minero, demostrando un valor actual neto atractivo.

- La empresa brindará un impacto social beneficioso al generar puestos de trabajo y otorgar valor agregado a materias primas nacionales.

- Se puede concientizar al mercado nacional en el uso de grasa lubricante con disulfuro de molibdeno en la industria en general, en armamento y vehículos.

- Es posible la extracción de gran variedad de productos y subproductos del disulfuro de molibdeno, los cuales son solicitados a nivel internacional.

\section{BIBLIOGRAFÍA}

Adex (2010). "Importaciones y exportaciones del periodo 2003-2009". Data Trade. Base de datos. Lima: Universidad de Lima [Consulta: 23 de mayo del 2010.]

Alcalá Cruz, Edgar B.; Flores Corrales, Alfredo y Arturo Beltrán Alfonso (2006). Manual de entrenamiento en concentración de minerales. Servicio Nacional de Geología y Técnico de Minas (Sergeotecmin). Bolivia. 
Aranzabe, Estíbaliz y Adolfo Málaga (2004). "Grasas lubricantes”. Boletín Mensual sobre Lubricación y Mantenimiento 5. <http://www. wearcheckiberica.es/> [Consulta: 28 de noviembre del 2009.]

Bankrate. Rates, interest rates. Canadá: Libor. <http://www.bankrate. com/rates/interest-rates/libor.aspx>. [Consulta: 26 de octubre del 2010.]

Banco Central de Reserva del Perú (2005). "Mercado de molibdeno". Estudios Económicos 29. Lima.

Castrol Limited (1999-2011). Productos. Illinois. <http://www.castrol. com/industrial>. [Consulta: 3 de octubre del 2009.]

Compañía Minera Antamina. Nuestros productos. Lima. <http://www. antamina.com>. [Consulta: 23 de marzo del 2010.]

Cofide (2010). Programas y líneas de financiamiento. Lima. <http:// www.cofide.com.pe>. [Consulta: 25 de octubre del 2010].

Comité Perú. ISO 26000. Lima. <http://www.iso26000peru.org>. [Consulta: 17 de enero del 2011.]

Díaz Garay, Bertha; Jarufe Zedán, Benjamín y María Teresa Noriega Araníbar (2007). Disposición de planta. 2. ${ }^{a}$ edición. Lima: Universidad de Lima, Fondo Editorial.

Díaz, Juan José (2002). Ficha comercial de grasas. Gulf. <www.gulf.es/ es/content/NT00004AA2.pdf>. [Consulta: 2 de octubre del 2009.]

INEI (2007). Censos nacionales 2007: XI de Población y VI de Vivien$d a$. Lima. <http://www.inei.gob.pe>. [Consulta: 13 de marzo del 2010.]

INEI (2007). Censos nacionales de población y vivienda 1940, 1961, 1972, 1981, 1993 y 2007. Lima. <http://www.inei.gob.pe>. [Consulta: 13 de marzo del 2010.]

INEI (2002). "Informe técnico de abril del 2002". Resultados de la Encuesta Nacional de Hogares sobre Condiciones de Vida y Pobre$z a$. Lima. <http://www.inei.gob.pe>. [Consulta: 13 de marzo del 2010.]

Kallpa Securities. Inversiones en bolsa. Lima. <http://www.kallpasab. com> [Consulta: 17 de agosto del 2010.] 
Karavaiko, G. I. et al. (2000). "Microbial degradation of cyanide and thiocyanate”. Microbiology. Vol. 69, núm. 2. Moscú: MAIK Nauka/Interperiodica.

Loayza Suárez, Rosa Estela (2010). "Entrevista con funcionario de banca empresarial". Lima: Banco Continental. [Consulta: 2 febrero del 2011.]

Logsdon, Mark; Kagelstein, Karen y Mudder Terry (2003). El manejo del cianuro en la extracción del oro. Ontario: Consejo Internacional de Metales y Medio Ambiente.

Ministerio de Energía y Minas. "Estadística eléctrica". Lima. <http:// www.minem.gob.pe>. [Consulta: 5 de octubre del 2010.]

Ministerio de la Producción. "Industria, normas técnicas y supervisión industrial". Estadísticas del sector minero. Lima: <http://www. produce.gob.pe>. [Consulta: 21 de setiembre del 2010.]

Mott, Robert L. (2006). Diseño de elementos de máquinas. 4. ${ }^{a}$ Edición. México D. F.: Pearson Education/Prentice Hall.

Mundi Crespo, Eloy (1972). Los lubricantes y sus aplicaciones. Madrid: Interciencia.

Olguín Galarza, Luis Ramón (2007). Apuntes de la asignatura de Administración Financiera. Lima: Universidad de Lima.

Operativa aduanera. Lima: <http://www.aduanet.gob.pe>. [Consulta: 5 de octubre del 2010.]

Perera Aldama, Luis (2007). Hacia un cuarto estado financiero básico. Santiago de Chile: Price Waterhouse Coopers.

Sapag Chaín, Nassir (2007). Proyectos de inversión. Formulación y evaluación. México D. F.: Pearson Educación.

Sastre Noboa, José Antonio (2010). "Estudio de prefactibilidad para la implementación de una planta productora de grasa lubricante a base de disulfuro de molibdeno para el sector minero". Tesis para optar el título de ingeniero industrial. Lima: Universidad de Lima.

Sociedad Nacional de Minería, Petróleo y Energía (SNMPE) (2005). “El molibdeno". Informe quincenal de la SNMPE 16. Lima. 
Software Google. Google Earth. California. [Consulta: 3 de setiembre del 2008.]

Southern Copper Corporation. Nosotros, Productos, Molibdeno. Lima. $<$ http://www.southernperu.com>. [Consulta: 5 de setiembre del 2010.]

Sunass (2007). Indicadores promedio nacionales 2007. Lima. <http:// www.sunass.gob.pe>. [Consulta: 13 de marzo del 2010.]

Sunass (2007). Informe de supervisión y fiscalización EPS Tacna S. A. Lima. <http://www.sunass.gob.pe>. [Consulta: 13 de marzo del 2010.]

Tamn \& Libor (2010). Actualidad empresarial, indicadores. Lima. <http://www.aempresarial.com/web/tasa_activa_mn_me.php>. [Consulta: 26 de octubre del 2010.]

Tantaleán Vanini, Guillermo (2004). Recursos metalúrgicos: Metalurgia general II. Lima: Universidad Nacional Mayor de San Marcos, E. A. P. de Ingeniería Metalúrgica.

Todoexpertos. Foro de expertos en distintas materias. [blog]. Barcelona. <http://www.todoexpertos.com>. [Consulta: 9 de marzo del 2010.]

Toskano Hurtado, Gérard Bruno (2005). "El proceso de análisis jerárquico (AHP) como herramienta para toma de decisiones en la selección de proveedores: Aplicación en la selección del proveedor para la Empresa Gráfica Comercial MyE S.R.L.”. Tesis para optar el título de licenciado en Investigación Operativa. Lima: Universidad Nacional Mayor de San Marcos.

Valverde Martínez, Aniceto (1985). Fundamentos y técnicas de la lubricación. Madrid: Alción.

Wikipedia. <http://es.wikipedia.org/wiki/Portada>. [Consulta: 16 de setiembre del 2010.] 